\title{
Structure and spectroscopic properties of imine acetaldehyde: a possible interstellar molecule
}

\author{
Pilar Redondo, ${ }^{\star}$ Antonio Largo and Carmen Barrientos \\ Computational Chemistry Group, Departamento de Química Física y Química Inorgánica, Facultad de Ciencias, Universidad de Valladolid, 47011 \\ Valladolid, Spain
}

Accepted 2018 April 27. Received 2018 March 21; in original form 2018 April 24

\begin{abstract}
A previous theoretical study shows that imine acetaldehyde can be obtained from the reaction between protonated vinyl alcohol and azanone. Therefore, imine acetaldehyde could be considered as a good molecule candidate to be found in space and could evolve to more complex organic molecules of prebiotic interest. In this work, we carried out a computational study of the different conformers of imine acetaldehyde. To characterize its conformers we apply a composite approach which considers the extrapolation to the complete basis set limit and core-valence electron correlation corrections at the couplet clusters level including single and double excitations and a perturbative treatment of triple excitations. This approach provides bond distances with an accuracy of 0.001-0.002 $\AA$ and angles accurate to $0.05-0.1^{\circ}$. Vibrational harmonic and anharmonic frequencies and infrared intensities are also reported at the CCSD level. The most stable structure corresponds to an antiperiplanar disposition of the oxygen atom and of $\mathrm{NH}$ group with the hydrogen atom of the $\mathrm{NH}$ group addressed outside the skeleton. Interconversion processes between the four conformers characterized are studied. The lowest isomerization barrier is estimated to be around $1.2 \mathrm{kcal} \mathrm{mol}^{-1}$, making these processes unlikely under low-temperature conditions, such as those reigning in the interstellar medium. The reported, at 'spectroscopic' accuracy, stabilities, molecular structures, as well as spectroscopic parameters for the four imine acetaldehyde conformers that could help in their laboratory or astronomical detection.
\end{abstract}

Key words: astrobiology - astrochemistry - ISM: general-ISM: molecules.

\section{INTRODUCTION}

Almost 200 molecules have been detected, to date, in the interstellar medium (ISM) or circumstellar shells. Carbon and other biogenic elements such as nitrogen, oxygen, and hydrogen are some of the most abundant in the universe and a large proportion of the molecules identified in space contain some of these elements. Formamide, $\mathrm{NH}_{2} \mathrm{CHO}$, was the first compound found in the interstellar medium that contains $\mathrm{C}, \mathrm{N}, \mathrm{O}$, and $\mathrm{H}$ all in the same molecule. It was observed towards Sgr B2 and likely towards Sgr A in Rubin et al. (1971) and years later was reported the detection of related transitions (Gottlieb et al. 1973) and isotopic forms (Lazareff, Lucas \& Encrenaz 1978). Since these initial reports, several studies have been devoted to interstellar formamide (Halfen, Ilyushin \& Ziurys 2011; Motiyenko et al. 2012; Kahane et al. 2013; Coutens et al. 2016; Thiel et al. 2017). In addition to the detection of interstellar formamide, three isomers of isocyanic acid, HCNO (Snyder \& Buhl 1972; Brunken et al. 2009, 2010; Marcelino et al.

^E-mail: predondo@qf.uva.es
2009), and other relevant prebiotic molecules, containing $\mathrm{C}, \mathrm{N}$, $\mathrm{O}$, and $\mathrm{H}$, as acetamide (Hollis et al. 2006), cyanoformaldehyde (Remijan et al. 2008), urea (Remijan et al. 2014), methyl isocyanate (Halfen, Ilyushin \& Ziurys 2015; Cernicharo et al. 2016), and N-metylformamide (Belloche et al. 2017), have been also observed. In a parallel way, the interest in the possible processes of formation in the ISM of these molecules has grown considerably. To know their synthesis paths and the reactions between detected molecules helps to understand the evolution to more complex systems.

In this context, and in order to study the viability of formation of complex organic molecules (COMs) through reaction between detected species, we have recently studied the ion-molecule reaction between azanone, $\mathrm{HNO}$, and protonated vinyl alcohol, $\mathrm{CH}_{2} \mathrm{CHOH}_{2}^{+}$ (Redondo et al. 2017). Azanone or nitroxyl, was the first molecule with NO bond, detected in the ISM. It was observed in emission from the directions of Sgr B2 $(\mathrm{OH})$ and NGC 2024 in Ulich, Hollis \& Snyder (1977). Some years later, in 1991, the interstellar HNO identification was confirmed (Hollis et al. 1991) and, in addition, HNO was observed towards several molecular clouds (Snyder et al. 1993; Ziurys et al. (1994). Vinyl alcohol $\left(\mathrm{CH}_{2} \mathrm{CHOH}\right)$ was 
detected in emission towards Sagittarius B2 (N) by means of its millimeter-wave rotational transitions in Turner \& Apponi (2001). The proton affinity of vinyl alcohol is higher than that of azanone (Grandinetti et al. 1992; Fairley et al. 1996), and in both cases, is higher than proton affinity of $\mathrm{H}_{2}\left(\mathrm{H}_{3}^{+}\right.$is the most abundant protondonor species in dense interstellar clouds Lepp, Dalgarno \& Sternberg (1987)). Therefore, we considered the ion-molecule reaction between $\mathrm{CH}_{2} \mathrm{CHOH}_{2}^{+}$and $\mathrm{HNO}$. From this reaction 12 thermodynamically viable interstellar products were obtained (Redondo et al. 2017). Even though, $\mathrm{NH}_{2} \mathrm{CHCOOH}^{+}+\mathrm{H}_{2}$ and $\mathrm{NH}_{2} \mathrm{CH}_{2} \mathrm{CO}^{+}+\mathrm{H}_{2} \mathrm{O}$ were, in principle, the most favourable products (reaction enthalpies are -93.3 and $-81.3 \mathrm{kcal} \mathrm{mol}^{-1}$, respectively, at the $\operatorname{CCSD}(\mathrm{T}) /$ augcc-pVTZ//MP2/aug-cc-pVTZ level); paths giving cis- and trans$\mathrm{O}$-protonated imine acetaldehyde (cis- $\mathrm{NHCHCHOH}^{+}$and trans$\mathrm{NHCHCHOH}^{+}$) were the most plausible from both a thermodynamic (largest exothermicity) and a kinetic (lowest energy barrier) point of view. Trans-NHCHCHOH${ }^{+}$is $8.3 \mathrm{kcal} \mathrm{mol}^{-1}$ more stable than cis- $\mathrm{NHCHCHOH}^{+}$at the $\mathrm{CCSD}(\mathrm{T}) /$ aug-cc-pVTZ//MP2/augcc-pVTZ level and the conversion barrier from the cis conformer into the most stable trans one is only of $0.4 \mathrm{kcal} \mathrm{mol}^{-1}(\approx 200 \mathrm{~K})$. From these results, the main conclusion of the study (Redondo et al. 2017) was that imine acetaldehyde, the neutral counterpart of O-protonated imine acetaldehyde, could be a feasible candidate molecule to be searched for in space.

Imine acetaldehyde, $\mathrm{NHCHCHO}$, is an isomer of methyl isocyanate, $\mathrm{CH}_{3} \mathrm{NCO}$. Methyl isocyanate is one of the most abundant species detected on the comet surface. It was first identified in the $3 \mathrm{~mm}$ segment of a broad-band survey of Sgr B2(N) (Halfen et al. 2015 ) in 2015 and only one year later a rigorous detection of this important missing molecule in astrochemical networks interstellar was carried out (Cernicharo et al. 2016). Imine acetaldehyde contains $\mathrm{C}, \mathrm{N}, \mathrm{O}$, and $\mathrm{H}$, if it is present in the ISM, could evolve to the formation of prebiotic molecules.

The main aim of this study is to characterize imine acetaldehyde conformers at the so-called 'spectroscopic' accuracy. To obtain this accuracy, it is necessary sophisticated quantum chemical models and taking into account core-valence (CV) correlation contributions and extrapolation to the complete basis set (CBS) limit (Puzzarini \& Barone 2018 and references therein). When this level is not computationally affordable (for medium and large size molecules) composite approaches, which are based on the fact that basis set and $\mathrm{CV}$ correlation effects can be evaluated at low-computational levels, are employed. The composite approach $\mathrm{CCSD}(\mathrm{T}) / \mathrm{CBS}+\mathrm{CV}$ (Heckert, Kállay \& Gauss 2005; Heckert et al. 2006), employed in this work, considers the extrapolation to the CBS limit and CV corrections at the couplet clusters level including single and double excitations and a perturbative treatment of triple excitations, CCSD(T), level (Raghavachari et al. 1989). It is shown that this approach provides bond distances with an accuracy of $0.001-0.002 \AA$ and angles accurate to $0.05-0.1^{\circ}$. As an example, this approach has been employed to characterize the elusive glycine VIp conformer (Barone et al. 2013), they showed that the obtained accuracy at the composite level is similar to the best experimental determination for the most stable conformers.

In this work, we carried out a computational study of imine acetaldehyde low-lying conformers providing information about their stabilities, molecular structures, as well as spectroscopic parameters, which could help in their laboratory or astronomical detection. To the best of our knowledge, there have been no previous experimental or theoretical studies on this species.

\section{COMPUTATIONAL METHODS}

We have used several methodological approaches with different basis sets running from density functional theory (DFT) to coupled cluster (CC) calculations. Equilibrium structures of the different conformers of imine acetaldehyde were preliminary optimized at the DFT level using the B3LYP hybrid exchange correlation functional (Becke 1986, 1988a), which includes the Lee-Yang-Parr (Lee, Yang \& Parr 1988) correlation functional and the Becke hybrid exchange functional (Becke 1988b). Within this approach the triple split-valence 6-311+G(d,p) basis set of Pople Krishnan et al. (1980) was used. This basis set includes diffuse functions for carbon, nitrogen, and oxygen atoms, as well as polarization functions for both hydrogen and heavy atoms. Subsequent optimizations using the second order Moller-Plesset theory (MP2; Moller \& Plesset 1934) were carried out. In this case, the correlated consistent polarized valence triple- $\zeta$ augmented with diffuse function basis sets of Dunning, namely aug-cc-pVTZ (Dunning 1989; Kendall, Dunning $\&$ Harrison 1992) was employed.

We have also performed geometry optimizations at the CC level including single and double excitations (CCSD; Raghavachari et al. 1989) in conjunction with the standard triple- $\zeta$ correlatedconsistent cc-pVTZ (Dunning 1989) basis set of Dunning as well as the triple- $\zeta$ aug-cc-pVTZ (Dunning 1989; Kendall et al. 1992) basis set augmented by diffuse functions. CCSD calculations were performed assuming the frozen-core (fc) approximation. Thus to account for core-correlation effects, we have used the CV correlation-consistent cc-pCVTZ basis set (Dunning 1989; Woon \& Dunning 1995). To improve the electron-correlation treatment, the CCSD approximation augmented by a perturbative treatment of triple excitations, $\operatorname{CCSD}(\mathrm{T})$, (Raghavachari et al. 1989) method was also used together with the triple- and quadruple- $\zeta$ correlated-consistent basis sets of Dunning, cc-pVTZ, and cc-pVQZ (Dunning 1989).

Energetic data were computed using the same levels of theory as those employed in geometry optimizations. It should be noted that in the $\operatorname{CCSD}(\mathrm{T})$ calculations, we used the T1 diagnostic (Lee \& Taylor 1989) to check the possible multireference character. It was found to be below the 0.01 value (Martin et al. 1992) indicating that, in all cases, a multiconfigurational procedure was not necessary. In addition, the intrinsic reaction coordinate (IRC) formalism (Gonzalez \& Schelegel 1990) was used to check the connections between the transition-state structures and minima.

Harmonic vibrational frequencies were computed on the optimized geometries at the CCSD/cc-pVTZ level using analytic second derivatives of the energy. To help in the possible experimental detection of these conformers by infrared (IR) spectroscopy, we also computed anharmonic vibrational frequencies. Anharmonic corrections were calculated at the CCSD/cc-pVTZ level of theory within a second-order perturbation treatment (VPT2; Barone 2005) based on a full cubic force field (CFF) and semidiagonal quartic force constants. Vibration-rotation interaction constants were also evaluated from the $\mathrm{CFF}$ calculations, allowing for correction of rotational constants, including vibrational effects.

Geometrical parameters, equilibrium rotational constants, and electronic energies were refined through a composite procedure (Heckert et al. 2005, 2006; Huang \& Lee 2008). Within this scheme, assuming additivity, the different contributions are evaluated separately at the highest possible level. The estimated properties, namely, geometrical parameters, equilibrium constants, and electronic energies, computed applying the composite scheme, which we will 

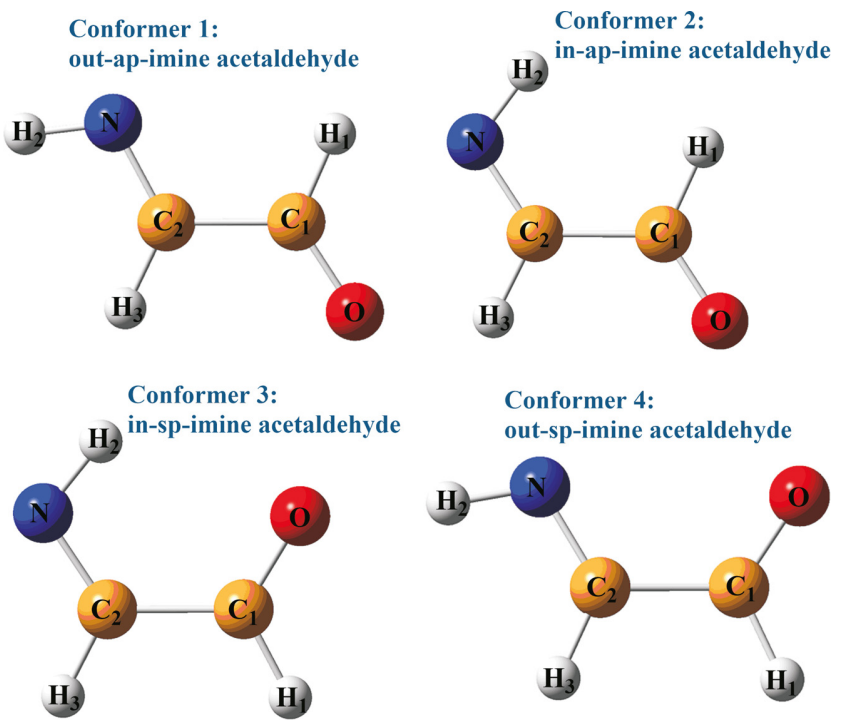

Figure 1. Structure of the four conformers located for imine acetaldehyde on the ${ }^{1} \mathrm{~A}^{\prime}$ potential energy surface

denote as $P(\operatorname{comp})$, are obtained using the following expression (Barone et al. 2013):

$P($ comp $)=P(\mathrm{CBS})+\Delta P(\mathrm{CV})+\Delta P($ aug $)$.

The first term, $P(\mathrm{CBS})$ accounts for basis-set truncation effects and corresponds to the CBS limit. It was evaluated through the $n^{-3}$ extrapolation formula (Helgaker et al. 1997) applied to the case $n=3$ and 4 , that is employing the $\operatorname{CCSD}(\mathrm{T}) / \mathrm{cc}-\mathrm{pVTZ}$ and $\operatorname{CCSD}(\mathrm{T}) / \mathrm{cc}-\mathrm{pVQZ}$ data. The second term, $\triangle P(\mathrm{CV})$, accounts for the $\mathrm{CV}$ electron correlation correction and is obtained by the following difference:

$\Delta P(\mathrm{CV})=P(\mathrm{CVTZ}$, all $)-P(\mathrm{CVTZ}, \mathrm{fc})$,

where $P(\mathrm{CVTZ}$, all $)$ and $P(\mathrm{CVTZ}, \mathrm{fc})$ correspond to the considered property evaluated at the CCSD/cc-pCVTZ level correlating all (full) and only valence electrons, (fc), respectively. The last term in equation (1), $\Delta P($ aug), accounts for the correction due to the inclusion of diffuse functions (aug) in the basis set and is computed as:

$\Delta P($ aug $)=P(\operatorname{augVTZ})-P(\mathrm{VTZ})$

where $P(\operatorname{augVTZ})$ and $P(\mathrm{VTZ})$ represent the considered property computed at the CCSD level using the aug-cc-pVTZ and cc-pVTZ basis sets, respectively, within the fc approximation.

All calculations were carried out with the GAUSSIAN 09 (Frisch at al. 2010) and CFOUR (Stanton et al. 2013) packages.

\section{RESULTS AND DISCUSSION}

We have found four low-lying conformers for imine acetaldehyde, which are collected in Fig. 1 . All of them have $\mathrm{C}_{s}$ symmetry with ${ }^{1} \mathrm{~A}^{\prime}$ electronic ground states. As can be seen in Fig. 1, in the conformer 1, the oxygen atom and the $\mathrm{NH}$ group lie on opposite sides of the plane, containing the $\mathrm{C}-\mathrm{C}$ bond, which is perpendicular to the molecular plane, and the hydrogen atom of the $\mathrm{NH}$ group is addressed outside the skeleton of the molecule (out). This conformation, taking as reference the $\mathrm{C}-\mathrm{C}$ bond, can be described as antiperiplanar (ap) and we denote as out-ap-imine acetaldehyde. Conformer 2 corresponds to the antiperiplanar conformer containing the hydrogen atom of the
Table 1. Relative energies, in $\mathrm{kcal} \mathrm{mol}^{-1}$, for the four conformers of imine acetaldehyde computed at different levels of theory (ZPV energies included).

\begin{tabular}{lcccc} 
& \multicolumn{3}{c}{ Conformer } & \multicolumn{3}{c}{ Conformer } & Conformer & Conformer \\
Level & 1 & 2 & 3 & 4 \\
\hline B3LYP/6-311+G(d,p) & 0.00 & 1.60 & 2.35 & 5.64 \\
MP2/aug-cc-pVTZ & 0.00 & 1.21 & 2.02 & 5.59 \\
CCSD/cc-pVTZ & 0.00 & 1.32 & 1.90 & 5.52 \\
CCSD/aug-cc-pVTZ & 0.00 & 1.41 & 2.33 & 5.70 \\
CCSD(Full)/cc-pCVTZ & 0.00 & 1.31 & 1.90 & 5.54 \\
CCSD(T)/cc-pVTZ & 0.00 & 1.10 & 1.57 & 5.35 \\
CCSD(T)/cc-pVQZ & 0.00 & 1.15 & 1.83 & 5.48 \\
Composite & 0.00 & 1.30 & 2.46 & 5.77 \\
\hline
\end{tabular}

$\mathrm{NH}$ group addressing in the direction of the skeleton of the molecule (in) and it is named as in-ap-imine acetaldehyde. Conformers 3 and 4 , with the oxygen atom and the $\mathrm{NH}$ group lying on the same side of the plane, containing the $\mathrm{C}-\mathrm{C}$ bond, which is perpendicular to the molecular plane, are described as synperiplanar ( $\mathrm{sp}$ ) when the $\mathrm{C}-\mathrm{C}$ bond is taken as reference. Of them, conformer 3, which includes the $\mathrm{H}$ atom of the $\mathrm{NH}$ group pointing in the direction of the main skeleton of the molecule, is denoted as in-sp-imine acetaldehyde. Finally, conformer 4, with the hydrogen atom of the $\mathrm{NH}$ group addressing outside the skeleton of the molecule, is named as outsp-imine acetaldehyde.

\subsection{Energetic}

The relative energies of the low-lying conformers (see Fig. 1) computed at different levels of theory are collected in Table 1. Antiperiplanar conformers, structures 1 and 2, are more stable than synperiplanar ones, structures 3 and 4 . Regardless of the level of theory used, the most stable conformer of imine acetaldehyde corresponds to structure 1. Following in energy is structure 2, which lies only $1.30 \mathrm{kcal} \mathrm{mol}^{-1}$ higher in energy than conformer 1 at composite level. Of the two synperiplanar conformers, structure 3, which includes the $\mathrm{H}$ atom of the $\mathrm{NH}$ group pointing in the direction of the main skeleton of the molecule, is located $2.46 \mathrm{kcal}$ $\mathrm{mol}^{-1}$ above the most stable conformer. Finally, structure 4 with the hydrogen atom of the $\mathrm{NH}$ group addressing outside the skeleton of the molecule lies $5.77 \mathrm{kcal} \mathrm{mol}^{-1}$ (at the composite level) above the lowest lying conformer. In the synperiplanar conformers, the interaction between the hydrogen atom of the group $\mathrm{NH}$ and the oxygen one favours the in structure, conformer 3, over the out one, conformer 4.

All different levels of theory used in this work provide the same stability order of conformers. The relative energies calculated at the different levels of theory are similar in all cases. However, it should be noted that at the MP2 level of theory, the $\mathrm{C}_{s^{-}}$symmetry conformer corresponding to structure 4 has an imaginary frequency of $54 \mathrm{i} \mathrm{cm} \mathrm{cm}^{-1}$ (a' symmetry) towards breaking out of the planar structure. We found a true minimum on the corresponding Potential Energy Surface (PES) with $\mathrm{C}_{1}$-symmetry $\left({ }^{1} \mathrm{~A}\right.$ electronic state) almost isoenergetic to the $\mathrm{C}_{s}$-conformer reflecting the extremely flatness of the PES along the out-of-plane mode. It should be pointed out that relative energies obtained at the B3LYP level (Table 1) for all conformers are similar to the composite ones.

In addition to the relative stability of the different conformers, it could be also important to study the possible interconversion processes between conformers. The transition states for the 


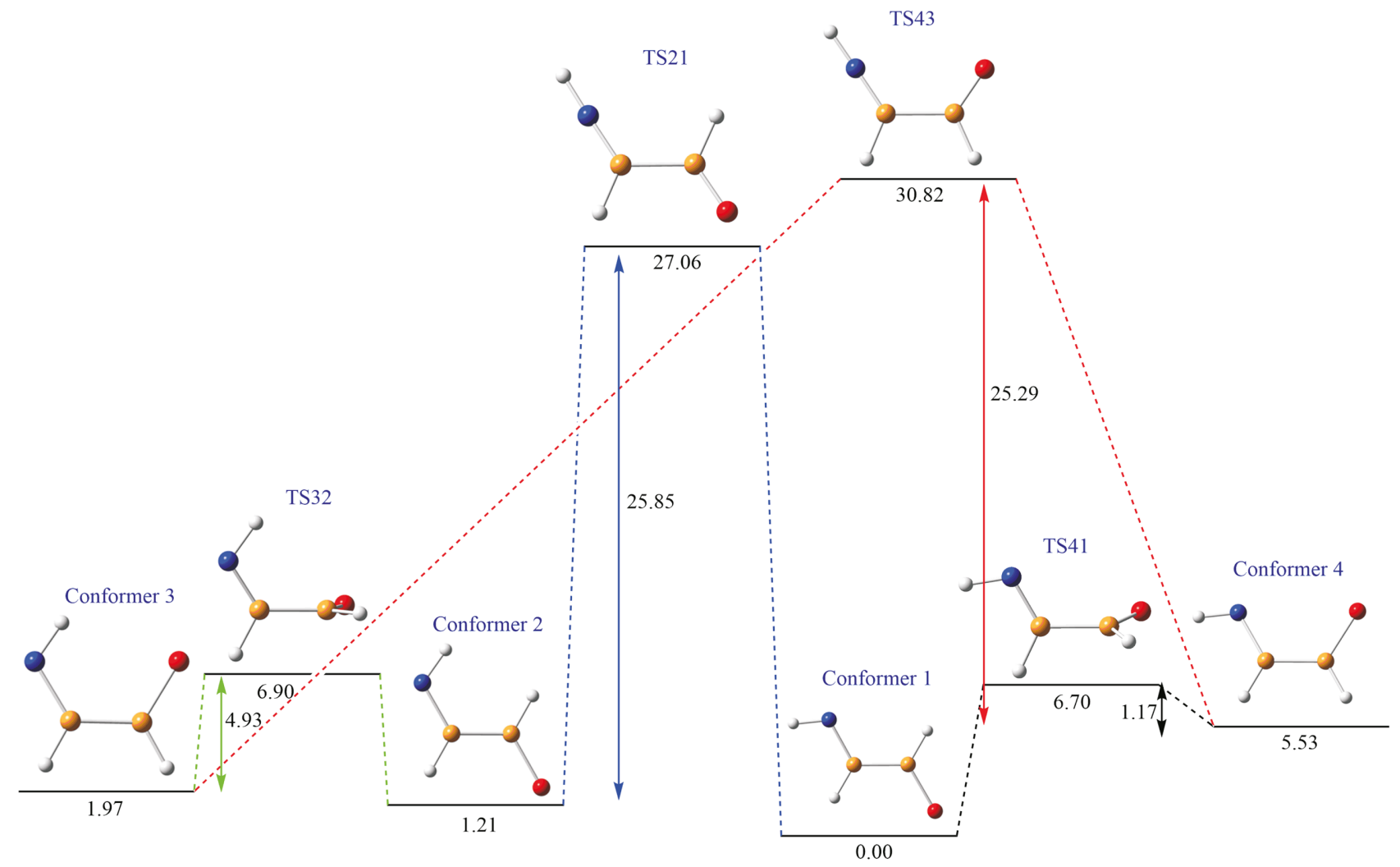

Figure 2. Interconversion barrier between the four conformers located for imine acetaldehyde. Relative energies, in $\mathrm{kcal}^{\mathrm{mol}}{ }^{-1}$, are calculated respect to conformer 1 at the CCSD(T)/aug-cc-pVQZ//CCSD/cc-pVTZ level (ZPV energies are included).

isomerization processes between conformers are depicted in Fig. 2. At the CCSD(T)/aug-cc-pVQZ//CCSD/cc-pVTZ level the barrier for the interconversion between the two most stable conformers, which only differs in the $\mathrm{CNH}$ angle, conformer $2 \longrightarrow$ conformer 1 , is about $25.85 \mathrm{kcal} \mathrm{mol}^{-1}(\approx 13008 \mathrm{~K})$. A similar barrier was found for the interconversion process between conformer $4 \longrightarrow$ conformer 3 which also differ in the $\mathrm{CNH}$ angle. In this case the transition state structure (TS43) connecting both conformers is located $25.29 \mathrm{kcal} \mathrm{mol}^{-1}(\approx 12726 \mathrm{~K})$ above conformer 4 . These high barriers indicate that both interconversion processes should have very slow rates. We also consider the interconversion process between synperiplanar $\leftrightarrow$ antiperiplanar conformations, which differ in the relative orientation of the oxygen atom, and the $\mathrm{NH}$ group. The interconversion barrier for the conformer $4 \longrightarrow$ conformer 1 process is $1.17 \mathrm{kcal} \mathrm{mol}^{-1}(\approx 589 \mathrm{~K})$ whereas for the inverse process is $6.70 \mathrm{kcal} \mathrm{mol}^{-1}(\approx 3372 \mathrm{~K})$. Hence, conformer 4 could more easily convert into the lowest lying conformer. In the case of the interconversion process between conformer $3 \longrightarrow$ conformer 2 , the corresponding transition state structure (TS32) lies $4.93 \mathrm{kcal}$ $\mathrm{mol}^{-1}(\approx 2481 \mathrm{~K})$ higher in energy than conformer 3 . This barrier suggests that the interconversion process between these two conformers should have a relatively slow rate. The transition states for the different interconversion processes, TS21, TS32, TS41, and TS43, computed at the B3LYP levels are located $24.78 \mathrm{kcal} \mathrm{mol}^{-1}$, $7.23 \mathrm{kcal} \mathrm{mol}^{-1}, 6.70 \mathrm{kcal} \mathrm{mol}^{-1}$, and $28.53 \mathrm{kcal} \mathrm{mol}^{-1}$, above the conformer 1, respectively. We can see that the B3LYP methodology gives a good agreement for relative energies of the conformers but isomerization barriers differs significantly with respect to these calculations at the $\operatorname{CCSD}(\mathrm{T})$ level.

\subsection{Structure}

The equilibrium geometries for the imine acetaldehyde conformers obtained at the CCSD/cc-pVTZ and composite levels are given in Table 2. Geometrical parameters of the transition state structures for interconversion processes between conformers, computed at the CCSD/cc-pVTZ level, are also included in Table 2. All the confomers display similar structural characteristics, and these similarities are much more pronounced between the two conformations either with an antiperiplanar disposition (conformers 1 and 2 ) or with synperiplanar orientation (conformers 3 and 4). In all the conformers, the $\mathrm{CO}$ bond lengths are very close to the typical C-O double bond value (1.205 Ain formaldehyde Gurvich; Veyts \& Alcock 1989), $1.216 \AA$ in acetaldehyde (Hollenstien \& Gunthard 1971). The C-N bond distances values are compatible with a typical C-N double bond (i.e. 1.273 $\AA$ in methanimine; Harmony et al. 1979). For all the isomers, the computed $\mathrm{C}-\mathrm{C}$ bond distances and the $\mathrm{CCO}$ angles are not far from the corresponding ones found in acetaldehyde (Hollenstien \& Gunthard 1971; $1.501 \AA$ and $123.9^{\circ}$, respectively).

With regard to the geometrical parameters for the transition state structures, we observe that, as expected, for the TS21 and TS43 structures, the $\mathrm{HNC}$ angle value $\left(178.50^{\circ}\right.$ and $179.34^{\circ}$, respectively, at the CCSD/cc-pVTZ level) is intermediate between those of the conformers that connect the transition state structures. In a similar way, for the transition state structures that connect synperiplanar and antiperiplanar conformations, namely TS32 and TS41, the dihedral angles $\left(\ngtr\left(\mathrm{NCCH}_{1}\right)=89.75^{\circ}\right.$ and $99.34^{\circ}$, respectively, at the CCSD/cc-pVTZ level) are in the middle point between those of the corresponding conformers $\left(0 .^{\circ}\right.$ and $180 .^{\circ}$, respectively). 
Table 2. Structural parameters (distances in Angstroms and angles in degrees) of the imine acetaldehyde conformers and the transition state structures.

\begin{tabular}{|c|c|c|c|c|c|c|c|c|c|}
\hline Parameter & Level & $\begin{array}{c}\text { Conformer } \\
1\end{array}$ & $\begin{array}{c}\text { Conformer } \\
2\end{array}$ & $\begin{array}{c}\text { Conformer } \\
3\end{array}$ & $\begin{array}{c}\text { Conformer } \\
4\end{array}$ & TS21 & TS32 & TS41 & TS43 \\
\hline $\mathrm{d}\left(\mathrm{O}-\mathrm{C}_{1}\right)$ & CCSD/cc-pVTZ & 1.2056 & 1.2032 & 1.2051 & 1.2015 & 1.2040 & 1.2049 & 1.2130 & 1.2016 \\
\hline \multirow[t]{2}{*}{$\mathrm{d}\left(\mathrm{C}_{1}-\mathrm{C}_{2}\right)$} & CCSD/cc-pVTZ & 1.4956 & 1.4988 & 1.5109 & 1.5105 & 1.5140 & 1.5131 & 1.5032 & 1.5294 \\
\hline & Composite & 1.4906 & 1.4930 & 1.5075 & 1.5070 & & & & \\
\hline $\mathrm{d}\left(\mathrm{N}-\mathrm{C}_{2}\right)$ & CCSD/cc-pVTZ & 1.2708 & 1.2706 & 1.2685 & 1.2691 & 1.2370 & 1.2680 & 1.2751 & 1.2321 \\
\hline $\mathrm{d}\left(\mathrm{C}_{1}-\mathrm{H}_{1}\right)$ & Composite & 1.1009 & 1.1067 & 1.1011 & 1.1039 & & & & \\
\hline \multirow[t]{2}{*}{$\mathrm{d}\left(\mathrm{C}_{2}-\mathrm{H}_{3}\right)$} & CCSD/cc-pVTZ & 1.0900 & 1.0861 & 1.0870 & 1.0924 & 1.1000 & 1.0947 & 1.0937 & 1.1044 \\
\hline & Composite & 1.0903 & 1.0867 & 1.0874 & 1.0925 & & & & \\
\hline \multirow[t]{2}{*}{$\mathrm{d}\left(\mathrm{N}-\mathrm{H}_{2}\right)$} & CCSD/cc-pVTZ & 1.0179 & 1.0228 & 1.0240 & 1.0183 & 0.9870 & 1.0248 & 1.0203 & 0.9865 \\
\hline & Composite & 1.0176 & 1.0227 & 1.0242 & 1.0178 & & & & \\
\hline$\ngtr\left(\mathrm{C}_{2} \mathrm{C}_{1} \mathrm{O}\right)$ & CCSD/cc-pVTZ & 122.62 & 123.43 & 121.87 & 124.07 & 122.80 & 122.99 & 122.58 & 123.64 \\
\hline$\ngtr\left(\mathrm{H}_{1} \mathrm{C}_{1} \mathrm{C}_{2}\right)$ & Composite & 114.98 & 114.90 & 116.96 & 114.91 & & & & \\
\hline \multirow[t]{2}{*}{$\ngtr\left(\mathrm{H}_{3} \mathrm{C}_{2} \mathrm{C}_{1}\right)$} & CCSD/cc-pVTZ & 116.04 & 116.36 & 116.73 & 117.50 & 113.47 & 116.34 & 116.35 & 113.18 \\
\hline & Composite & 116.55 & 116.69 & 117.52 & 115.55 & & & & \\
\hline \multirow[t]{3}{*}{$\ngtr\left(\mathrm{H}_{2} \mathrm{NC}_{2}\right)$} & CCSD/cc-pVTZ & 110.74 & 109.85 & 108.08 & 110.01 & 178.50 & 111.74 & 110.35 & 179.34 \\
\hline & Composite & 111.57 & 110.84 & 109.15 & 110.93 & & & & \\
\hline & CCSD/cc-pVTZ & 0.00 & 0.00 & 180.00 & 180.00 & 0.00 & 89.75 & 99.34 & 180.00 \\
\hline$\ngtr\left(\mathrm{NC}_{2} \mathrm{C}_{1} \mathrm{H}_{1}\right)$ & Composite & 0.00 & 0.00 & 180.00 & 180.00 & & & & \\
\hline
\end{tabular}

Table 3. Spectroscopic parameters for the imine acetaldehyde conformers (in $\mathrm{MHz})$ : Equilibrium $\left(\mathrm{A}_{e}, \mathrm{~B}_{e}\right.$, and $\left.\mathrm{C}_{e}\right)$ and ground vibrational state $\left(\mathrm{A}_{0}\right.$, $\mathrm{B}_{0}$, and $\mathrm{C}_{0}$ ) Rotational Constants are computed with the composite method and Centrifugal Distortion parameters in the Symmetrically Reduced Hamiltonian $\left(\mathrm{D}_{J}\right.$, $\mathrm{D}_{J K}, \mathrm{D}_{K}, \mathrm{~d}_{1}$, and $\mathrm{d}_{2}$ ) at the CCSD/cc-pVTZ level. Dipole Moments ( $\mu$ in Debye) are computed at the CCSD/cc-pVTZ level.

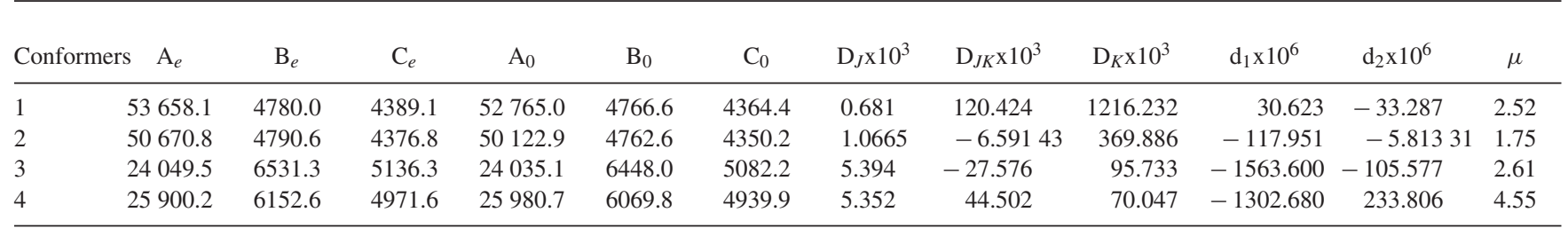

\subsection{Spectroscopic parameters}

The relevant spectroscopic parameters to rotational spectroscopy, together with the dipole moments for the imine acetaldehyde conformers are collected in Table 3. The values of equilibrium rotational constants were obtained from the bond distances computed at the composite level showed in Table 2. The corresponding constants for the ground vibrational state were computed from vibration-rotation coupling constants and degeneracy factors for the vibrational modes from anharmonic vibrational frequencies at the CCSD/cc-pVTZ level. The similarities found in structural parameters between the two conformations either with an antiperiplanar disposition (conformers 1 and 2) or with synperiplanar orientation (conformers 3 and 4) are reflected in the calculated rotational constants. We can also see in Table 3 that all the conformers have relative high dipole moments $(1.75-4.55 \mathrm{D})$ that should favour their possible detection provided they can be formed in the ISM.

The predicted harmonic and anharmonic vibrational frequencies and the corresponding IR intensities, computed at the CCSD/ccpVTZ level, for the imine acetaldehyde conformers, are given in Table 4. Convergence problems are found in the calculation of anharmonic contribution at the CCSD/cc-pVTZ level for the conformer 1 , and in this case, the anharmonic contributions are computed at the MP2/cc-pVTZ level. This information could help for an eventual detection in the gas phase of imine acetaldehyde through IR spectroscopy. An intense band associated with the $\mathrm{C}-\mathrm{O}$ stretching mode dominates the IR spectrum of all conformers. This band is located at $1798 \mathrm{~cm}^{-1}, 1815 \mathrm{~cm}^{-1}, 1799 \mathrm{~cm}^{-1}$, and $1812 \mathrm{~cm}^{-1}$ in conformers $1,2,3$, and 4 , respectively.

As usual, the comparison between obtained harmonic and anharmonic frequencies shows, in general, that harmonic frequencies are slightly higher than the corresponding anharmonic ones and the absolute differences are more important in the larger frequencies corresponding to stretching modes. The only exception is the negative anharmonicity found in some bending modes as the $\mathrm{a}^{\prime}$ NCC-bending mode of conformer 1 located at $344 \mathrm{~cm}^{-1}$. Regarding IR intensities there are no straightforward variations when passing from the harmonic to the anharmonic approach. This shows that it is not possible to found a general scale factor to obtain IR anharmonic intensities from harmonic ones. Therefore, to describe correctly the intensities of the IR spectrum is necessary to take into account anharmonic contributions. 
Table 4. CCSD/cc-pVTZ Harmonic, $\omega$, and Anharmonic, $v$, Vibrational Frequencies $\left(\mathrm{cm}^{-1}\right)$ and IR Intensities, $\mathrm{I}_{\text {har }}$ and $\mathrm{I}_{\text {anhar }}\left(\mathrm{km} \mathrm{mol}{ }^{-1}\right)$, for the imine acetaldehyde conformers.

\begin{tabular}{|c|c|c|c|c|c|c|c|c|c|c|c|c|c|c|c|c|}
\hline \multirow[b]{2}{*}{ Mode } & \multicolumn{4}{|c|}{ Conformer $1^{a}$} & \multicolumn{4}{|c|}{ Conformer 2} & \multicolumn{4}{|c|}{ Conformer 3} & \multicolumn{4}{|c|}{ Conformer 4} \\
\hline & $\omega$ & $\mathrm{I}_{\text {har }}$ & $v$ & $\mathrm{I}_{\text {anhar }}$ & $\omega$ & $\mathrm{I}_{\text {har }}$ & $v$ & $\mathrm{I}_{\text {anhar }}$ & $\omega$ & $\mathrm{I}_{\text {har }}$ & $v$ & $\mathrm{I}_{\text {anhar }}$ & $\omega$ & $\mathrm{I}_{\text {har }}$ & $v$ & $\mathrm{I}_{\text {anhar }}$ \\
\hline$a^{\prime}$ & 3503 & 2.1 & 3337 & 0.3 & 3434 & 1.2 & 3270 & 1.4 & 3433 & 2.9 & 3280 & 3.4 & 3496 & 1.0 & 3329 & 0.3 \\
\hline$a^{\prime}$ & 3121 & 20.4 & 2954 & 26.0 & 3173 & 8.7 & 3052 & 7.2 & 3155 & 26.9 & 2990 & 5.7 & 3085 & 52.7 & 2919 & 42.0 \\
\hline$a^{\prime}$ & 3017 & 50.3 & 2863 & 46.8 & 2951 & 73.5 & 2812 & 69.0 & 3003 & 87.1 & 2862 & 83.6 & 2967 & 99.0 & 2847 & 50.6 \\
\hline$a^{\prime}$ & 1831 & 103.9 & 1798 & 91.3 & 1833 & 111.2 & 1815 & 357.0 & 1826 & 66.8 & 1799 & $* * *$ & 1841 & 76.0 & 1812 & 263.3 \\
\hline$a^{\prime}$ & 1715 & 22.3 & 1683 & 17.2 & 1712 & 17.8 & 1680 & 13.7 & 1714 & 14.3 & 1683 & 6.2 & 1723 & 7.7 & 1691 & 8.1 \\
\hline$a^{\prime}$ & 1426 & 25.3 & 1393 & 20.0 & 1421 & 8.6 & 1386 & 22.8 & 1458 & 7.5 & 1432 & 6.0 & 1458 & 22.4 & 1426 & 19.9 \\
\hline$a^{\prime}$ & 1389 & 13.2 & 1354 & 15.9 & 1412 & 23.7 & 1369 & 0.0 & 1416 & 22.2 & 1387 & 17.7 & 1417 & 7.1 & 1389 & 7.1 \\
\hline$a^{\prime}$ & 1243 & 36.8 & 1220 & 37.2 & 1254 & 25.9 & 1227 & 28.3 & 1257 & 90.9 & 1237 & 36.1 & 1228 & 49.5 & 1207 & 50.0 \\
\hline$a^{\prime}$ & 1145 & 6.6 & 1116 & 6.5 & 1160 & 61.2 & 1134 & 60.1 & 1176 & 62.9 & 1146 & 61.1 & 1142 & 6.6 & 1116 & 6.4 \\
\hline$a^{\prime}$ & 1058 & 4.5 & 1037 & 3.5 & 1074 & 58.5 & 1046 & 57.7 & 1057 & 0.2 & 1039 & 0.3 & 1051 & 7.5 & 1034 & 8.4 \\
\hline$a^{\prime}$ & 1052 & 26.0 & 1026 & 26.4 & 1047 & 0.4 & 1030 & 0.6 & 913 & 36.0 & 894 & 31.3 & 915 & 21.8 & 896 & 25.0 \\
\hline$a^{\prime}$ & 717 & 61.3 & 690 & 58.1 & 730 & 10.5 & 793 & 259.7 & 790 & 25.2 & 770 & 21.2 & 775 & 72.9 & 804 & 23.8 \\
\hline$a^{\prime}$ & 588 & 1.5 & 579 & 1.4 & 578 & 2.6 & 569 & 2.3 & 700 & 10.7 & 682 & 11.1 & 655 & 46.9 & 624 & 36.8 \\
\hline$a^{\prime}$ & 344 & 37.6 & 345 & 36.0 & 342 & 37.0 & 346 & 35.7 & 305 & 18.3 & 295 & 16.5 & 297 & 0.2 & 297 & 0.2 \\
\hline$a^{\prime}$ & 155 & 19.0 & 154 & 18.3 & 145 & 16.9 & 144 & 16.1 & 189 & 10.2 & 181 & 10.1 & 53 & 2.4 & 104 & 0.9 \\
\hline
\end{tabular}

Note. ${ }^{a}$ Anharmonic contributions for conformer 1 are computed at the MP2/cc-pVTZ level.

\section{CONCLUSIONS}

We have carried out a theoretical study of imine acetaldehyde conformers by means of different quantum chemical approaches. The composite level employed allows us to obtain equilibrium structures, rotational parameters, and relative energies with 'spectroscopic' accuracy. Vibrational harmonic and anharmonic frequencies and intensities have been computed at the CCSD level.

Four low-lying conformers have been characterized on the ${ }^{1} \mathrm{~A}^{\prime}$ PES. The most stable conformer of imine acetaldehyde corresponds to structure 1, out-ap-imine acetaldehyde. In this conformer, the oxygen atom and the $\mathrm{NH}$ group lie on opposite sides of the plane, containing the $\mathrm{C}-\mathrm{C}$ bond, which is perpendicular to the molecular plane, and the hydrogen atom of the $\mathrm{NH}$ group is addressed outside the skeleton of the molecule (out). Conformer 2 with the hydrogen atom of the $\mathrm{NH}$ group addressing in the direction of the skeleton of the molecule (in) lies only $1.30 \mathrm{kcal} \mathrm{mol}^{-1}$ higher in energy than conformer 1 at composite level. The two synperiplanar conformers, structures 3 (in) and 4 (out), with the oxygen atom and the NH group lying on the same side of the plane, are located $2.46 \mathrm{kcal} \mathrm{mol}^{-1}$ and $5.77 \mathrm{kcal} \mathrm{mol}^{-1}$ above conformer 1, respectively, at the composite level.

We have also analysed the interconversion processes between conformers. The more easier process corresponds to the isomerization of conformer 4 (the less stable) to conformer 1 (the most stable) with an interconversion barrier of $1.17 \mathrm{kcal} \mathrm{mol}^{-1}(\approx 589 \mathrm{~K})$. These results show that at the low temperatures of the interstellar medium the four conformers could coexist.

The relevant spectroscopic parameters to rotational spectroscopy and vibrational frequencies are reported for the four conformers. This information could help in their laboratory or astronomical detection by radioastronomy or IR spectroscopy.

\section{ACKNOWLEDGEMENTS}

Financial support from the Spanish Ministerio de Economía Industria y Competitividad (Grant AYA2017-87515-P). This work is a result of the collaboration of the COST Action TD 1308.

\section{REFERENCES}

Barone V., 2005, J. Chem. Phys., 122, 014108

Barone V., Biczysko M., Bloino J., Puzzarini C., 2013a, Phys. Chem. Chem. Phys., 15, 1358

Barone V., Biczysko M., Bloino J., Puzzarini C., 2013b, J. Chem. Theory Comput., 9, 1533

Becke A. D., 1986, J. Chem. Phys., 84, 4524

Becke A. D., 1988a, J. Chem. Phys., 88, 2547

Becke A. D., 1988b, J. Chem. Phys., 88, 1053

Belloche A. et al., 2017, A\&A, 601, A49

Brunken S., Gottlieb C. A., McCarthy M. C., Thaddeus P., 2009, ApJ, 697, 880

Brunken S., Belloche A., Martín S., Verheyen L., Menten K. M ., 2010, A\&A, 516, A109

Cernicharo J. et al., 2016, A\&A, 587, L4

Coutens A. et al., 2016, A\&A, 590, L6

Dunning T. H., 1989, J. Chem. Phys, 90, 1007

Fairley D. A., Scott G. B. I., Freeman C. G., Maclagan R. G. A. R., McEwan M. J., 1996, J. Chem. Soc. Faraday Trans., 92, 1305

Frisch M. J. et al., 2010, Gaussian 09, Gaussian Inc, Wallingford CT

Gonzalez C., Schelegel H. B., 1990, J. Phys. Chem., 94, 5523

Gottlieb C. A., Palmer P., Richard L. J., Zuckerman B., 1973, ApJ, 182, 699

Grandinetti F., Hrusak J., Schroder D., Schwarz H., 1992, J. Phys. Chem., 96, 2100

Gurvich L. V., Veyts I. V., Alcock C. B., 1989, Thermodynamic Properties of Individual Substances. Hemisphere Pub. Co., New York

Halfen D. T., Ilyushin V. V., Ziurys L. M., 2011, ApJ, 743, 60

Halfen D. T., Ilyushin V. V., Ziurys L. M., 2015 ApJ, 812, L5

Harmony M. D., Laurie V. W., Kuczkowski R. L., Schwendeman R. H., Ramsay D. A., Lovas F. J., Lafferty W. J., Maki A. G., 1979, J. Phys. Chem. Ref. Data, 8, 619

Heckert M., Kállay M., Gauss J., 2005, Mol. Phys., 103, 2109

Heckert M., Kállay M., Tew D. P., Klopper W., Gauss J., 2006, J. Chem. Phys., 125, 044108

Helgaker T., Klopper W., Koch H., Noga J., 1997, JChPh, 106, 9639

Hollenstien H., Gunthard, Hs H., 1971, Spec. Acta, 27A, 2027

Hollis J. M., Snyder L. E., Ziurys L. M., McGonagle D., 1991, in Haschick A. D., Ho P. T. P., eds., ASP Conf. Ser., Vol. 16, Atoms, Ions and Molecules: New Results in Spectral Line Astrophysics, Astron. Soc. Pac., San Francisco. , 407

Hollis J. M., Lovas F. J., Remijan A. J., Jewell P. R., Ilyushin V. V., Kleiner I., 2006, ApJ, 643, L25

Huang X., Lee T. J., 2008, J. Chem. Phys., 129, 044312 
Kahane C., Ceccarelli C., Faure A., Caux E., 2013, ApJ, 763, L38

Kendall R. A., Dunning T. H., Harrison R. J., 1992, J. Chem. Phys., 96, 6796

Krishnan R., Binkley J. S., Seeger R., Pople J. A., 1980, J. Chem. Phys., 72, 650

Lazareff B., Lucas R., Encrenaz P., 1978, A\&A, 70, L77

Lee T. J., Taylor P. R., 1989, Int. J. Quantum Chem., 36, 199

Lee C., Yang W., Parr R. G., 1988, Phys. Rev. B , 37, 785

Lepp S., Dalgarno A., Sternberg A., 1987, ApJ, 321, 383

Marcelino N., Cernicharo J., Tercero B., Roueff E., 2009, ApJ, 690, L27

Martin J. M. L., Lee T. J., Scuseria G. E., Taylor P. R., 1992, J. Chem. Phys., 97, 6549

Moller C., Plesset M., 1934, PhRv., 46, 618

Motiyenko R. A., Tercero B., Cernicharo J., Margules L., 2012, A\&A, 548, A71

Puzzarini C., Barone V., 2018, Acc. Chem. Res., 51, 548

Raghavachari K., Trucks G. W., Pople J. A., Head-Gordon M., 1989, Chem. Phys. Lett., 157, 479

Redondo P., Martínez H., Largo A., Barrientos C., 2017, A\&A, 603, A139
Remijan A. J., Hollis J. M., Lovas F. J., Stork W. D., Jewell P. R., Meier D. S., 2008, ApJ, 675, L85

Remijan A. J. et al., 2014, ApJ, 783, 77

Rubin R. H., Swenson G. W., Jr, Solomon R. C., Flygare H. L., 1971, ApJ, 169, L39

Snyder L. E., Buhl D., 1972, ApJ, 177, 619

Snyder L. E., Kuan Y. J., Ziurys L. M., Hollis J. M., 1993, ApJ, 403, L17

Stanton J. F., Gauss J., Harding M. E., Szalay P. G., 2013, CFOUR, A Quantum Chemical Program Package

Thiel V., Belloche A., Menten K. M., Garrod R. T., Muller H. S. P., 2017, A\&A, 605, L6

Turner B. E., Apponi A. J., 2001, ApJ, 561, L207

Ulich B. L. J., Hollis J. M., Snyder L. E., 1977, ApJ, 217, L105

Woon D. E., Dunning T. H., Jr, 1995, J. Chem. Phys., 103, 4572

Ziurys L. M., Hollis J. M., Snyder L. E., 1994, ApJ, 430, 706

This paper has been typeset from a $\mathrm{T}_{\mathrm{E}} \mathrm{X} / \mathrm{LT} \mathrm{E} \mathrm{X}$ file prepared by the author 


\section{List of astronomical key words (Updated on 2017 March)}

This list is common to Monthly Notices of the Royal Astronomical Society, Astronomy and Astrophysics, and The Astrophysical Journal. In order to ease the search, the key words are subdivided into broad categories. No more than six subcategories altogether should be listed for a paper.

The subcategories in boldface containing the word 'individual' are intended for use with specific astronomical objects; these should never be used alone, but always in combination with the most common names for the astronomical objects in question. Note that each object counts as one subcategory within the allowed limit of six.

The parts of the key words in italics are for reference only and should be omitted when the keywords are entered on the manuscript.

\section{General}

editorials, notices

errata, addenda

extraterrestrial intelligence

history and philosophy of astronomy

miscellaneous

obituaries, biographies

publications, bibliography

sociology of astronomy

standards

\section{Physical data and processes}

acceleration of particles

accretion, accretion discs

asteroseismology

astrobiology

astrochemistry

astroparticle physics

atomic data

atomic processes

black hole physics

chaos

conduction

convection

dense matter

diffusion

dynamo

elementary particles

equation of state

gravitation

gravitational lensing: micro

gravitational lensing: strong

gravitational lensing: weak

gravitational waves

hydrodynamics

instabilities

line: formation

line: identification

line: profiles

magnetic fields

magnetic reconnection

(magnetohydrodynamics) MHD

masers

molecular data

molecular processes

neutrinos

nuclear reactions, nucleosynthesis, abundances

opacity

plasmas

polarization radiation: dynamics

radiation mechanisms:general

radiation mechanisms: non-thermal

radiation mechanisms: thermal

radiative transfer

relativistic processes

scattering

shock waves

solid state: refractory

solid state: volatile

turbulence

waves

\author{
Astronomical instrumentation, methods and techniques \\ atmospheric effects \\ balloons \\ instrumentation: adaptive optics \\ instrumentation: detectors \\ instrumentation: high angular resolution \\ instrumentation: interferometers \\ instrumentation: miscellaneous \\ instrumentation: photometers \\ instrumentation: polarimeters \\ instrumentation: spectrographs \\ light pollution \\ methods: analytical \\ methods: data analysis \\ methods: laboratory: atomic \\ methods: laboratory: molecular \\ methods: laboratory: solid state \\ methods: miscellaneous \\ methods: numerical \\ methods: observational \\ methods: statistical \\ site testing \\ space vehicles \\ space vehicles: instruments \\ techniques: high angular resolution \\ techniques: image processing \\ techniques: imaging spectroscopy \\ techniques: interferometric \\ techniques: miscellaneous \\ techniques: photometric \\ techniques: polarimetric \\ techniques: radar astronomy \\ techniques: radial velocities \\ techniques: spectroscopic \\ telescopes
}


Astronomical data bases

astronomical data bases: miscellaneous atlases

catalogues

surveys

virtual observatory tools

\section{Astrometry and celestial mechanics}

astrometry

celestial mechanics

eclipses

ephemerides

occultations

parallaxes

proper motions

reference systems

time

\section{The Sun}

Sun: abundances

Sun: activity

Sun: atmosphere

Sun: chromosphere

Sun: corona

Sun: coronal mass ejections (CMEs)

Sun: evolution

Sun: faculae, plages

Sun: filaments, prominences

Sun: flares

Sun: fundamental parameters

Sun: general

Sun: granulation

Sun: helioseismology

Sun: heliosphere

Sun: infrared

Sun: interior

Sun: magnetic fields

Sun: oscillations

Sun: particle emission

Sun: photosphere

Sun: radio radiation

Sun: rotation

(Sun:) solar-terrestrial relations

(Sun:) solar wind

(Sun:) sunspots

Sun: transition region

Sun: UV radiation

Sun: X-rays, gamma-rays

\section{Planetary systems}

comets: general

comets: individual: ...

Earth

interplanetary medium

Kuiper belt: general

Kuiper belt objects: individual: . . . meteorites, meteors, meteoroids minor planets, asteroids: general

minor planets, asteroids: individual: . . .
Moon

Oort Cloud

planets and satellites: atmospheres

planets and satellites: aurorae

planets and satellites: composition

planets and satellites: detection

planets and satellites: dynamical evolution and stability

planets and satellites: formation

planets and satellites: fundamental parameters

planets and satellites: gaseous planets

planets and satellites: general

planets and satellites: individual: ...

planets and satellites: interiors

planets and satellites: magnetic fields

planets and satellites: oceans

planets and satellites: physical evolution

planets and satellites: rings

planets and satellites: surfaces

planets and satellites: tectonics

planets and satellites: terrestrial planets

planet-disc interactions

planet-star interactions

protoplanetary discs

zodiacal dust

\section{Stars}

stars: abundances

stars: activity

stars: AGB and post-AGB

stars: atmospheres

(stars:) binaries (including multiple): close

(stars:) binaries: eclipsing

(stars:) binaries: general

(stars:) binaries: spectroscopic

(stars:) binaries: symbiotic

(stars:) binaries: visual

stars: black holes

(stars:) blue stragglers

(stars:) brown dwarfs

stars: carbon

stars: chemically peculiar

stars: chromospheres

(stars:) circumstellar matter

stars: coronae

stars: distances

stars: dwarf novae

stars: early-type

stars: emission-line, Be

stars: evolution

stars: flare

stars: formation

stars: fundamental parameters

(stars:) gamma-ray burst: general

(stars:) gamma-ray burst: individual: ...

stars: general

(stars:) Hertzsprung-Russell and colour-magnitude

diagrams

stars: horizontal branch

stars: imaging

stars: individual: . . .

stars: interiors 
stars: jets

stars: kinematics and dynamics

stars: late-type

stars: low-mass

stars: luminosity function, mass function

stars: magnetars

stars: magnetic field

stars: massive

stars: mass-loss

stars: neutron

(stars:) novae, cataclysmic variables

stars: oscillations (including pulsations)

stars: peculiar (except chemically peculiar)

(stars:) planetary systems

stars: Population II

stars: Population III

stars: pre-main-sequence

stars: protostars

(stars:) pulsars: general

(stars:) pulsars: individual: ...

stars: rotation

stars: solar-type

(stars:) starspots

stars: statistics

(stars:) subdwarfs

(stars:) supergiants

(stars:) supernovae: general

(stars:) supernovae: individual: ...

stars: variables: Cepheids

stars: variables: Scuti

stars: variables: general

stars: variables: RR Lyrae

stars: variables: S Doradus

stars: variables: T Tauri, Herbig Ae/Be

(stars:) white dwarfs

stars: winds, outflows

stars: Wolf-Rayet

Interstellar medium (ISM), nebulae

ISM: abundances

ISM: atoms

ISM: bubbles

ISM: clouds

(ISM:) cosmic rays

(ISM:) dust, extinction

ISM: evolution

ISM: general

(ISM:) HII regions

(ISM:) Herbig-Haro objects

ISM: individual objects: . . .

(except planetary nebulae)

ISM: jets and outflows

ISM: kinematics and dynamics

ISM: lines and bands

ISM: magnetic fields

ISM: molecules

(ISM:) photodissociation region (PDR)

(ISM:) planetary nebulae: general

(ISM:) planetary nebulae: individual: ...

ISM: structure

ISM: supernova remnants
The Galaxy

Galaxy: abundances

Galaxy: bulge

Galaxy: centre

Galaxy: disc

Galaxy: evolution

Galaxy: formation

Galaxy: fundamental parameters

Galaxy: general

(Galaxy:) globular clusters: general

(Galaxy:) globular clusters: individual: ...

Galaxy: halo

Galaxy: kinematics and dynamics

(Galaxy:) local interstellar matter

Galaxy: nucleus

(Galaxy:) open clusters and associations: general

(Galaxy:) open clusters and associations: individual: ...

(Galaxy:) solar neighbourhood

Galaxy: stellar content

Galaxy: structure

\section{Galaxies}

galaxies: abundances

galaxies: active

(galaxies:) BL Lacertae objects: general

(galaxies:) BL Lacertae objects: individual: ... .

galaxies: bulges

galaxies: clusters: general

galaxies: clusters: individual: ... .

galaxies: clusters: intracluster medium

galaxies: distances and redshifts

galaxies: dwarf

galaxies: elliptical and lenticular, $\mathrm{cD}$

galaxies: evolution

galaxies: formation

galaxies: fundamental parameters

galaxies: general

galaxies: groups: general

galaxies: groups: individual: ...

galaxies: haloes

galaxies: high-redshift

galaxies: individual: ...

galaxies: interactions

(galaxies:) intergalactic medium

galaxies: irregular

galaxies: ISM

galaxies: jets

galaxies: kinematics and dynamics

(galaxies:) Local Group

galaxies: luminosity function, mass function

(galaxies:) Magellanic Clouds

galaxies: magnetic fields

galaxies: nuclei

galaxies: peculiar

galaxies: photometry

(galaxies:) quasars: absorption lines

(galaxies:) quasars: emission lines

(galaxies:) quasars: general 
(galaxies:) quasars: individual: ...

(galaxies:) quasars: supermassive black holes galaxies: Seyfert

galaxies: spiral

galaxies: starburst

galaxies: star clusters: general

galaxies: star clusters: individual: ... .

galaxies: star formation

galaxies: statistics

galaxies: stellar content

galaxies: structure

\section{Cosmology}

(cosmology:) cosmic background radiation (cosmology:) cosmological parameters

(cosmology:) dark ages, reionization, first stars

(cosmology:) dark energy

(cosmology:) dark matter

(cosmology:) diffuse radiation

(cosmology:) distance scale

(cosmology:) early Universe

(cosmology:) inflation

(cosmology:) large-scale structure of Universe

cosmology: miscellaneous

cosmology: observations

(cosmology:) primordial nucleosynthesis

cosmology: theory

\author{
Resolved and unresolved sources as a function of \\ wavelength \\ gamma-rays: diffuse background \\ gamma-rays: galaxies \\ gamma-rays: galaxies: clusters \\ gamma-rays: general \\ gamma-rays: ISM \\ gamma-rays: stars \\ infrared: diffuse background \\ infrared: galaxies \\ infrared: general \\ infrared: ISM \\ infrared: planetary systems \\ infrared: stars \\ radio continuum: galaxies \\ radio continuum: general \\ radio continuum: ISM \\ radio continuum: planetary systems \\ radio continuum: stars \\ radio continuum: transients \\ radio lines: galaxies \\ radio lines: general \\ radio lines: ISM \\ radio lines: planetary systems \\ radio lines: stars \\ submillimetre: diffuse background \\ submillimetre: galaxies \\ submillimetre: general \\ submillimetre: ISM \\ submillimetre: planetary systems \\ submillimetre: stars \\ ultraviolet: galaxies
}

ultraviolet: general

ultraviolet: ISM

ultraviolet: planetary systems

ultraviolet: stars

X-rays: binaries

X-rays: bursts

X-rays: diffuse background

$\mathrm{X}$-rays: galaxies

$\mathrm{X}$-rays: galaxies: clusters

X-rays: general

X-rays: individual: ... .

X-rays: ISM

X-rays: stars 